DOI: https://doi.org/10.11144/Javeriana.umed59-2.savd

\title{
Síndrome de acueducto vestibular dilatado: a propósito de un caso
}

\author{
Enlarged Vestibular Aqueduct Syndrome: A Case Report
}

Recepción: 13/04/2015 | Aceptación: 15/11/2017

\author{
Gina Lorena Marín Gallego ${ }^{a}$ \\ Pontificia Universidad Javeriana, Colombia \\ Sebastián Barragán Delgado \\ Pontificia Universidad Javeriana, Colombia \\ Santiago Hernández González \\ Hospital Universitario San Ignacio, Colombia
}

a Correspondencia: ginamarin82@hotmail.com

\section{RESUMEN}

El acueducto vestibular dilatado es el hallazgo más encontrado en imágenes radiológicas de pacientes con hipoacusia neurosensorial $(1,2)$. La frecuencia del síndrome de acueducto vestibular dilatado continúa siendo subestimada, situación que ha llevado a subdiagnosticar pacientes con esta condición. El propósito del reporte de caso y su discusión es aclarar los aspectos más importantes del diagnóstico clínico, audiológico e imagenológico de esta patología, así como considerarla parte del diagnóstico diferencial de pacientes en estudio de hipoacusia.

Palabras clave

acueducto vestibular; pérdida auditiva conductiva; pérdida auditiva sensorineural.

\begin{abstract}
Large vestibular aqueduct is the most frequent condition found in radiological imaging of patients with sensorineural hearing loss. The frequency of this syndrome continues to be underestimated, which has lead to underdiagnosis. The purpose of this case report and its discussion is to clarify the most important aspects of the clinical diagnosis, audiology and radiology, as well as rise attention to the importance of this entity as part of the differential diagnosis in hearing loss workup.

Keywords

vestibular aqueduct; hearing loss; sensorineural; hearing loss; conductive.
\end{abstract}

\section{Reporte de caso}

El caso corresponde a una mujer de 34 años de edad, quien desde el periodo preescolar ha presentado episodios de otitis media aguda recurrente. Hasta el momento de la consulta, había recibido múltiples tratamientos antibióticos y, adicionalmente, miringotomía, con colocación de tubos de ventilación bilateral a los 7 años de edad.

Desde el período preescolar comenzó también con deterioro auditivo bilateral, de predominio en el oído derecho. En varias oportunidades, a la paciente le realizaron estudios
Cómo citar: Marín Gallego GL, Barragán Delgado S, Hernández González S. Síndrome de acueducto vestibular dilatado: a propósito de un caso. Univ. Med. 2018; 59(2). doi: https://doi.org/10.11144/ Javeriana.umed59-2.savd 
Gina Lorena Marín Gallego, Sebastián Barragán Delgado, Santiago Hernández González.

audiológicos que confirmaron diferentes grados de hipoacusia en el oído derecho, y de característica fluctuante (los estudios en orden de realización) (figuras 1,2 y 3 ).

Figura 1.Audiometría con evidencia de hipoacusia neurosensorial derecha severa

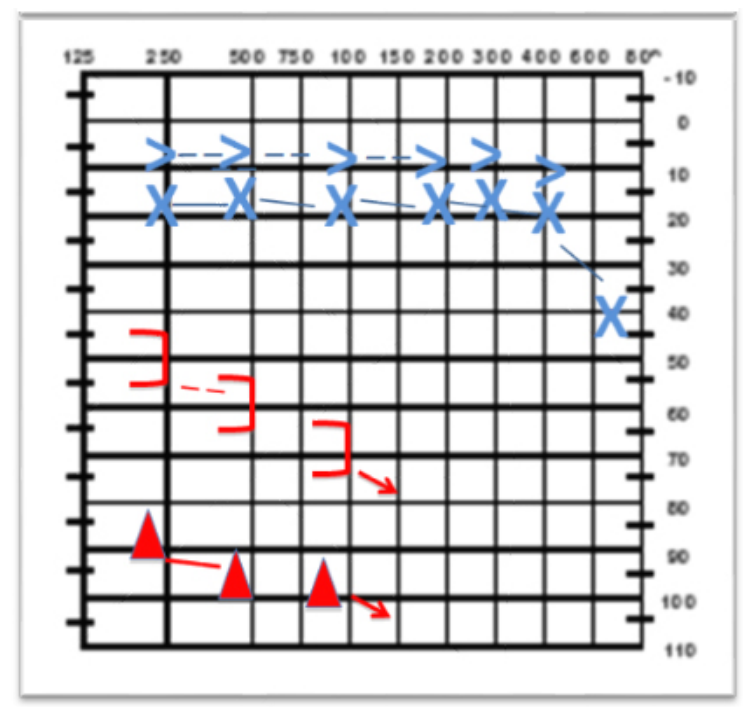

Figura 2.Audiometría con evidencia de hipoacusia mixta con gap aéreo-óseo en frecuencias graves y agudas

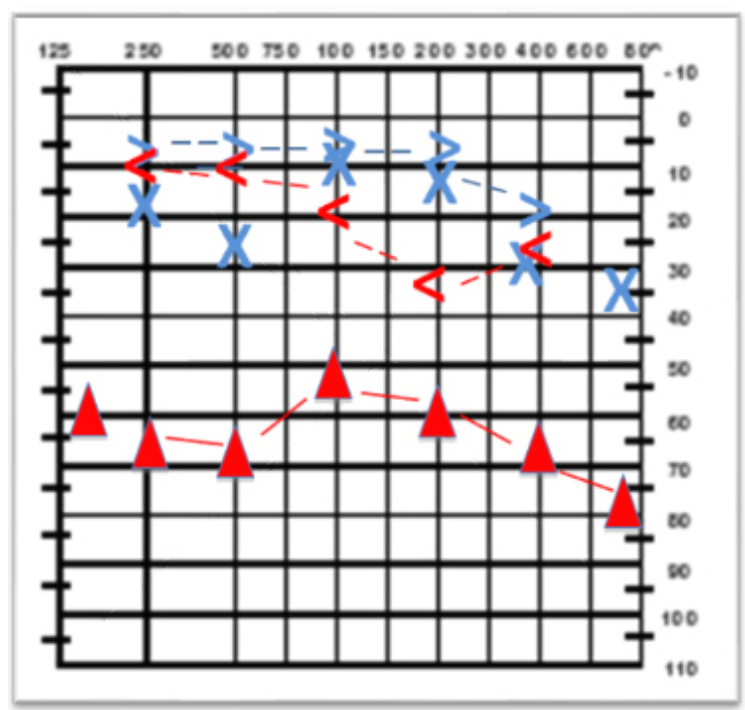

Figura 3.Audiometría con evidencia de hipoacusia neurosensorial profunda derecha

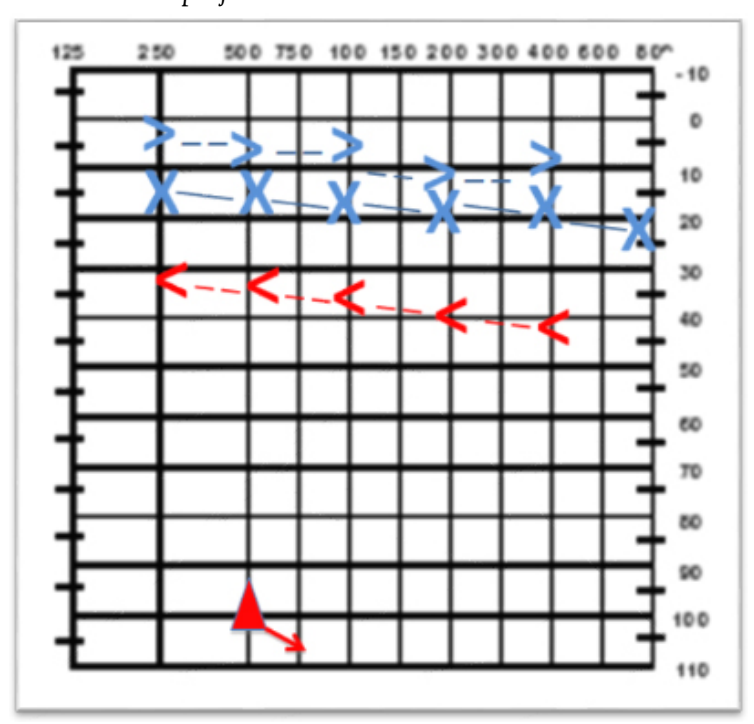

El médico tratante interpretó la hipoacusia de la paciente como una alteración auditiva causada por infecciones de oído a repetición. La audición se deteriora lentamente hasta cofosis derecha e hipoacusia neurosensorial de moderada a severa del oído izquierdo $(1,2)$.

En consulta neurootológica se solicita tomografía axial computarizada simple de oídos (figura 4) y resonancia nuclear magnética contrastada (figura 5). Se encontraron hallazgos compatibles con acueducto vestibular dilatado derecho. La paciente negó tener antecedente de trauma craneoencefálico y no desea rehabilitación con audífono.

Figura 4.Tomografía axial computarizada de oído. Se observa dilatación anormal de acueducto vestibular derecho

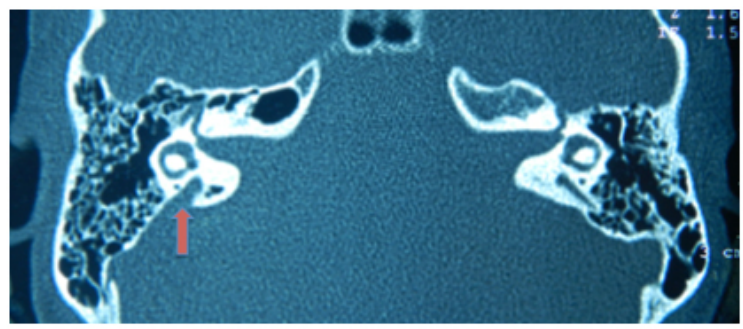


Figura 5.Resonancia magnética nuclear de oído con evidencia de imagen hiperintensa correspondiente a conducto endolinfático dentro del acueducto vestibular dilatado derecho

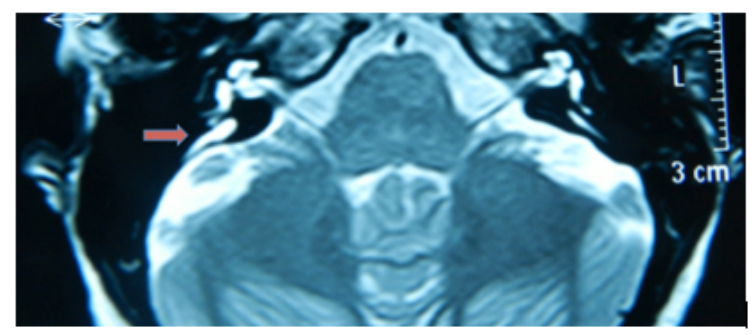

\section{Discusión}

El acueducto vestibular (AV) hace parte del hueso temporal; se origina en el oído interno, específicamente en el vestíbulo, para dirigirse hacia la fosa craneal posterior en el borde posterior del hueso petroso. Contiene el ducto endolinfático, que termina como saco endolinfático en la zona del opérculo óseo (3).

El acueducto vestibular dilatado (AVD) es una malformación del hueso temporal relacionada con hipoacusia en la niñez. Es el hallazgo más encontrado mediante técnicas de imagen en pacientes a quienes se les estudia hipoacusia neurosensorial congénita $(1,2,4)$. En 1791, Carlo Mondini describió por primera vez el síndrome durante la disección del hueso temporal de un niño con sordera congénita. En 1968, G. E. Valvassori y J. D. Clemis describieron por primera vez los hallazgos radiológicos del AVD y su relación con la hipoacusia neurosensorial congénita o adquirida tempranamente. En su estudio de 3700 tomografías se encontró la patología en 50 casos. Los autores consideraron un AV mayor a 1,5 mm de diámetro anteroposterior en tomografía axial computarizada como criterio de anormalidad. Esta condición se ha reportado más frecuentemente en las mujeres que en los hombres, con una relación 3:2 (5).

$\mathrm{Al}$ síndrome de AVD pueden asociarse otras anomalías del oído interno, como canales semicirculares dilatados, cóclea hipoplásica y vestíbulo dilatado, siendo el porcentaje de asociación muy variable entre los estudios publicados. En 1978, Valvassori reportó 50 pacientes con esta patología, al encontrar entre ellos anomalías adicionales en el 60\% de los casos. Becker (1983), Emmett (1985), Davidson (1999) y Atkin (2009) encontraron anomalías combinadas en un $41 \%, 88 \%, 86 \%$ y $78,4 \%$, respectivamente en, pacientes con acueducto vestibular dilatado.

Se ha descrito el AVD sindrómico y el no sindrómico. Para el primer grupo se ha encontrado asociación con trastornos genéticos como el síndrome de Pendred, el de Alagille, el branquiotorrenal, y con la asociación CHARGE (6). El síndrome de Pendred es la causa sindrómica más frecuente de acueducto vestibular dilatado. En 1996, el gen responsable de síndrome de Pendred fue mapeado en el cromosoma 7q31. Mutaciones de este gen (SLC26A4), que codifica para la proteína transmembranal relacionada con el transporte de cloro, yodo y bicarbonato expresada en el oído interno, glándula tiroides y riñón, pueden causar también hipoacusia no sindrómica recesiva, lo que sugiere una amplia heterogenicidad del alelo. El acueducto vestibular dilatado también ha sido relacionado con acidosis tubular renal, hipoacusia mixta congénita ligada a X, síndrome otofaciocervical, de Waardenburg y de Noonan, entre otros $(7,8)$.

Los síntomas vestibulares y auditivos hacen parte del cuadro clínico de los pacientes con acueducto vestibular dilatado y también son motivo de controversia en las diferentes publicaciones encontradas, especialmente en lo relacionado con el tipo y la progresión de la hipoacusia, así como de los factores precipitantes para su desarrollo (3).

La mayoría de estudios reportados coinciden en que la presentación bilateral es la más común, con amplios rangos de diferencia que llegan hasta el $87 \%$ de los casos $(1,2,3,6,9)$.

$\mathrm{Si}$ bien esta patología es un trastorno congénito, la edad inicial de presentación puede variar ampliamente y relacionarse con eventos específicos. La mayoría de artículos consideran el trauma craneoencefálico como uno de los factores precipitantes más encontrados, aunque este evento puede no ser de gran severidad para justificar el desarrollo de la 
hipoacusia. Algunos reportes indican hasta un $80 \%$ de incidencia de trauma craneoencefálico como factor precipitante (10); sin embargo, los rangos varían ampliamente y están afectados por los números limitados de casos reportados en la literatura. De hecho, uno de los estudios retrospectivos con mayor número de casos publicados (en total 77 casos de AVD) reporta solo un $4 \%$ relacionados con trauma craneoencefálico (1). Otros desencadenantes encontrados incluyen maniobra de Valsalva, barotrauma, infecciones respiratorias superiores, trauma acústico, ejercicio físico y fiebre (3).

Entender la variabilidad de presentación en el tipo de hipoacusia es importante para ofrecer el manejo apropiado a estos pacientes. La más frecuentemente reportada es la hipoacusia neurosensorial con severidad desde leve a profunda. Se ha hipotetizado que su presentación se debe a un incremento en la presión endolinfática, que daña las células cocleares por medio de un proceso inflamatorio, así como la asociación de partición incompleta, a menudo encontrada, y mutaciones genéticas específicas.

También existe un número significativo de pacientes que presentan un gap aéreo-óseo en frecuencias graves; más significativo este en frecuencias de 250 y $500 \mathrm{~Hz}$ y de un promedio de $22 \mathrm{~dB}(1,2,3,9,11)$. Otros reportes coinciden con estos hallazgos $(6,9)$. Un dato importante es que la mayoría de pacientes con gap aéreoóseo tienen, además, timpanogramas y reflejos estapediales normales $(1,6,9)$, por lo que se descarte la patología de oído medio como causa de la hipoacusia conductiva o mixta. Muchos de estos pacientes son llevados a procedimientos que buscan tratar patologías de oído medio, como timpanostomía con colocación de tubos de ventilación sin mejoría de la hipoacusia y, en algunos casos, con deterioro de esta.

Si bien no está aún clara la etiología de este gap, una de las teorías más recientes reconoce el acueducto vestibular dilatado como una forma de tercera ventana similar a la dehiscencia del canal semicircular superior, donde la energía acústica generada por la vía aérea se disipa por esta nueva tercera ventana, disminuyendo el umbral aéreo. Por el contrario, el umbral óseo aumenta debido a la disminución en la impedancia de la escala vestibular (por la presencia del acueducto vestibular dilatado), que aumenta la diferencia de presión entre las escalas y, en consecuencia, el movimiento la membrana basilar (9).

Ha sido también tema de debate las características de progresión de la hipoacusia. Cohortes retrospectivas (12) reportan audición estable en el $67 \%$ de 39 oídos estudiados con AVD; de estos el $34 \%$ presentaron fluctuación en su audición. La progresión de la pérdida auditiva se dio en el $33 \%$ de los oídos, de los cuales el 50\% demostraron fluctuación (6). Estudios más recientes reportan estabilidad solo en el 47\% de los oídos evaluados (7). La incidencia global de síntomas vestibulares es variable con reportes del $12 \%$ al $71 \%$, dependiendo de los estudios. Los más comunes incluyen episodios de vértigo severo, imbalance e incoordinación $(6,7,8)$. Otros síntomas otológicos son variables e incluyen acúfeno y sensación de plenitud aural.

La confirmación diagnóstica de esta entidad se hace a través de estudios de imagenología. La tomografía axial computarizada es la más utilizada para este objetivo; sin embargo, todavía se respecto a los criterios imagenológicos para hacer el diagnóstico. Existen dos puntos donde más frecuentemente se determina el diámetro del acueducto: el punto medio entre el vestíbulo y la apertura en la fosa craneal posterior y el punto donde se abre el opérculo externo. La propuesta inicial realizada por Valvassori y Clemis es la de un acueducto de 1,5 $\mathrm{mm}$ o mayor en el punto medio del trayecto del acueducto (3). Otras propuestas se muestran en la tabla 1.

Tabla 1.Propuestas para determinar el diámetro del acueducto

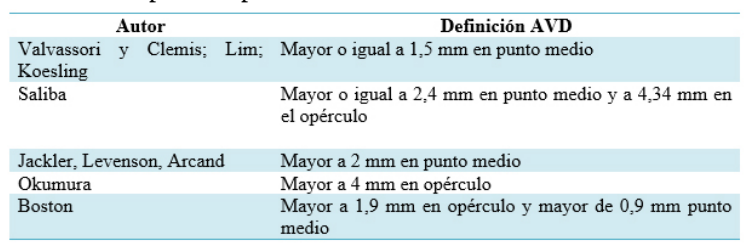

Dentro de las mediciones establecidas entre los diferentes grupos de investigación en nuestra institución, usamos la del grupo de Cincinnati, es decir, un AV de más de 0,9 mm en el punto medio y más de 1,9 mm en el opérculo. Este es el primer 
estudio en determinar la distribución normal del ancho del acueducto vestibular mediante tomografía en un grupo de niños con hipoacusia neurosensorial, comparado con los hallazgos en un grupo control sin hipoacusia. El estudio determina el percentil 95 de las medidas de control como el límite superior normal del ancho del acueducto vestibular por dos razones. La primera es que dentro de la distribución de valores encontrados del ancho del acueducto solo los valores superiores incluyen los anormales. La segunda razón es que previamente los acueductos con ancho en el punto medio entre $1,0 \mathrm{y}$ $1,4 \mathrm{~mm}$, que eran clasificados como borderline, tenían también una tendencia de hipoacusia neurosensorial progresiva.

En el anterior estudio otro aspecto destacado fue que si bien en la mayoría de pacientes con AVD la medición anormal se registró en el punto medio y en el opérculo, también existieron pacientes con la anormalidad solo en una de las dos medidas. En algunos pacientes con hipoacusia neurosensorial congénita el AVD es detectado con tomografía axial computarizada; sin embargo, esta no revela estructuras anatómicas del laberinto membranoso, por lo que se sugiere ser complementada con resonancia magnética nuclear para poder visualizar estos espacios con fluidos del laberinto, particularmente la porción extraósea del saco endolinfático, no valorable en la tomografía y que también podría estar dilatada (6).

Otros de los beneficios de la resonancia magnética es la menor exposición a radiación, especialmente para la población pediátrica, y la mejor visualización de las estructuras membranosas del oído interno, que podrían presentar malformaciones asociadas. Por lo anterior, y a pesar de que el estudio imagenológico de elección continúa siendo la tomografía de oído, recientes investigaciones se han enfocado en la utilidad de la resonancia magnética de alta resolución, que ha demostrado altas tasas de concordancia con la tomografía (88\%).

Ninguna de estas investigaciones ha evidenciado superioridad de un estudio sobre el otro. Por lo tanto, la decisión final del estudio imagenológico que se va a elegir debe ser individualizada y tener en cuenta la edad del paciente, características de su hipoacusia, costos, disponibilidad de tecnología y riesgos potenciales de cada estudio.

No existe en la actualidad un protocolo de manejo para estos pacientes; pero cada vez más se reportan con éxito pacientes con hipoacusias neurosensoriales rehabilitados con implante coclear.

Bajo el principio de que un proceso inflamatorio que afecta irreversiblemente las células ciliadas hace parte de la fisiopatología de esta condición, existen publicaciones que evalúan la respuesta al tratamiento con corticoides y resultados favorables (13). Hay que considerar, sin embargo, la naturaleza fluctuante de la hipoacusia, que ya ha sido reportada como una de las principales dificultades para dar validez a dichos estudios.

Los beneficios del implante coclear en estos pacientes ya han sido reportados por autores como Harker y colaboradores (14), e incluyen mejor desempeño para la discriminación del lenguaje, al comparar los pacientes antes de la rehabilitación con el implante y después de este. Deben considerarse las anomalías asociadas del oído interno dentro del planeamiento quirúrgico, especialmente el riesgo de "Gusher". En general, este no representa una contraindicación para realizar el procedimiento y los reportes de implante en estos pacientes no han mostrado mayor riesgo o complicaciones quirúrgicas (5).

\section{Conclusión}

El abordaje diagnóstico de los pacientes que presentan hipoacusia debe incluir el síndrome de AVD dentro de las posibilidades diagnósticas, especialmente si es de tipo neurosensorial, fluctuante y progresivo. Es preciso indagar factores desencadenantes de la hipoacusia; en el entretanto, el diagnóstico imagenológico debe incluir tomografía axial computarizada, donde se compruebe un diámetro del acueducto mayor a $1,9 \mathrm{~mm}$ a nivel del opérculo o mayor a 0,9 $\mathrm{mm}$ en el punto medio entre el vestíbulo y la apertura en la fosa craneal posterior. 
También es mandatorio considerar la realización de resonancia nuclear magnética como parte del estudio integral del paciente. En la actualidad, el manejo está orientado a la rehabilitación auditiva, de acuerdo con la severidad de la hipoacusia. Aquí el implante coclear es uno de los recursos más importantes para la rehabilitación del paciente con progresión de la enfermedad a grados profundos de pérdida auditiva.

\section{Referencias}

1. Govaerts PJ, Casselman J, Daemers K, De Ceulaer G, Somers T, Offeciers FE. Audiological findings in large vestibular aqueduct syndrome. Int $\mathrm{J}$ Pediatr Otorhinolaryngol. 1999 Dec 15;51(3):157-64.

2. Marques SR, Ajzen S, D Ippolito G, Alonso $\mathrm{L}$, Isotani S, Lederman $\mathrm{H}$. Morphometric analysis of the internal auditory canal by computed tomography imaging. Iran J Radiol Q J Publ Iran Radiol Soc. 2012 Jun;9(2):71-8.

3. Valvassori GE, Clemis JD. The large vestibular aqueduct syndrome. The Laryngoscope. 1978 May;88(5):723-8.

4. Wu C-C, Chen Y-S, Chen P-J, Hsu CJ. Common clinical features of children with enlarged vestibular aqueduct and Mondini dysplasia. The Laryngoscope. 2005 Jan;115(1):132-7.

5. Asma A, Anouk H, Luc VH, Brokx JPL, Cila U, Van De Heyning P. Therapeutic approach in managing patients with large vestibular aqueduct syndrome (LVAS). Int J Pediatr Otorhinolaryngol. 2010 May;74(5):474-81.

6. Berrettini S, Forli F, Bogazzi F, Neri E, Salvatori L, Casani AP, et al. Large vestibular aqueduct syndrome: audiological, radiological, clinical, and genetic features. Am J Otolaryngol. 2005 Dec;26(6):363-71.

7. Gopen Q, Zhou G, Whittemore K, Kenna M. Enlarged vestibular aqueduct: review of controversial aspects. The Laryngoscope. 2011 Sep;121(9):1971-8.

8. Zhou G, Gopen Q, Kenna MA. Delineating the hearing loss in children with enlarged vestibular aqueduct. The Laryngoscope. 2008 Nov;118(11):2062-6.

9. Vijayasekaran S, Halsted MJ, Boston M, Meinzen-Derr J, Bardo DME, Greinwald $\mathrm{J}$, et al. When is the vestibular aqueduct enlarged? A statistical analysis of the normative distribution of vestibular aqueduct size. AJNR Am J Neuroradiol. 2007 Jul;28(6):1133-8.

10. Santos S, Sgambatti L, Bueno A, Albi G, Suárez A, Domínguez MJ. [Enlarged vestibular aqueduct syndrome: A review of 55 paediatric patients]. Acta Otorrinolaringológica Esp. 2010 Oct;61(5):338-44.

11. Madden C, Halsted M, Benton C, Greinwald J, Choo D. Enlarged vestibular aqueduct syndrome in the pediatric population. Otol Neurotol Off Publ Am Otol Soc Am Neurotol Soc Eur Acad Otol Neurotol. $2003 \mathrm{Jul} ; 24(4): 625-32$.

12. Mori T, Westerberg BD, Atashband S, Kozak FK. Natural history of hearing loss in children with enlarged vestibular aqueduct syndrome. J Otolaryngol-Head Neck Surg J Oto-Rhino-Laryngol Chir Cervico-Faciale. 2008 Feb;37(1):112-8.

13. Grimmer JF, Hedlund G, Park A. Steroid treatment of hearing loss in enlarged vestibular aqueduct anomaly. Int J Pediatr Otorhinolaryngol. 2008 Nov;72(11):1711-5.

14. Harker LA, Vanderheiden S, Veazey D, Gentile N, McCleary E. Multichannel cochlear implantation in children with large vestibular aqueduct syndrome. Ann Otol Rhinol Laryngol Suppl. 1999 Apr;177:39-43. 